\title{
THE ROLE OF MICROSTRUCTURAL PHENOMENA IN MAGNETIC THIN FILMS
}

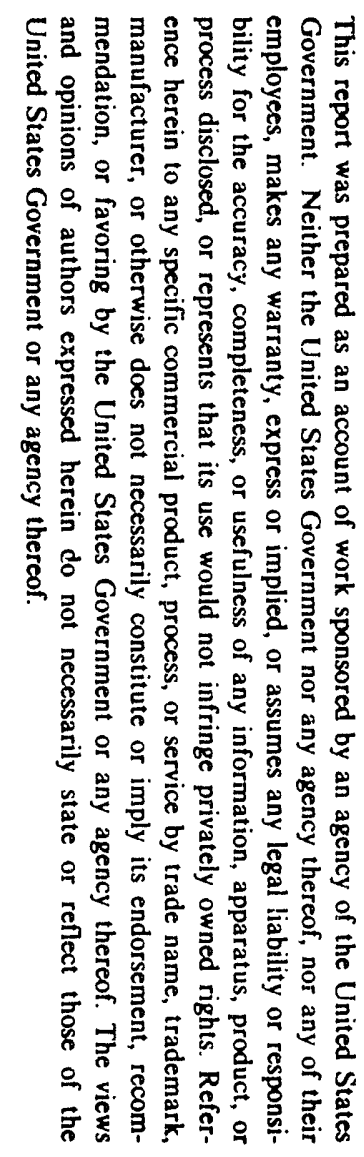

\author{
DEPARTMENT OF ENERGY
}

Attention: Dr. Michael Kassner

Germantown, MD 20874

FROM

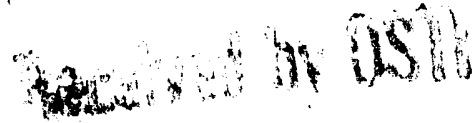

\section{CARNEGIE MELLON UNIVERSITY}

David E. Laughlin

\section{Department of Materials Science and Engineering}

David N. Lambeth

Department of Electrical and Computer Engineering Pittsburgh, Pennsylvania 15213

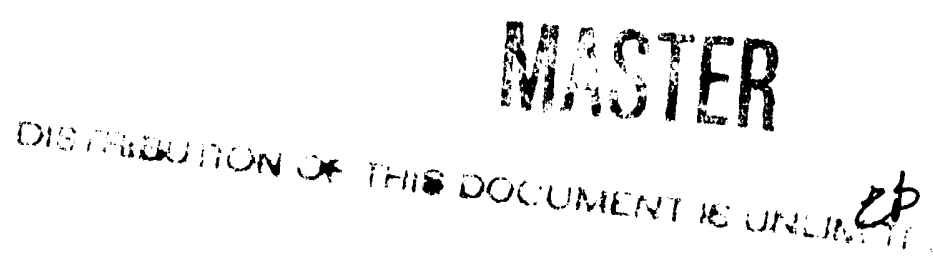




\section{Table of Contents}

$\begin{array}{llr} & \text { Abstract } & 3 \\ \text { I } & \text { Introduction } & 4 \\ \text { II } & \text { Review of Results to Date } & 6 \\ \text { III } & \text { Proposed Research } & 12 \\ \text { IV } & \text { Summary } & 16 \\ \text { V } & \text { References } & 17 \\ \text { VI } & \text { Publications and Oral Presentations } & 19 \\ \text { VII } & \text { Research Facilities at CMU } & 20 \\ \text { VIII } & \text { Personnel } & 25 \\ \text { IX } & \text { Budget } & 32 \\ \text { X } & \text { Current and Pending Support } & 48\end{array}$




\begin{abstract}
We summarize our results during the first two years of our research program. These results have helped us to document important features of the microstructure of magnetic thin films which influence their magnetic properties. During the next phase of the research we plan to isolate some of these features so that we may determine directly their effect on magnetic properties. The plans for our research are outlined in the proposed research section of this document. We also include a listing of our publications and oral presentations based on the work to date, followed by a description of the facilities we have available for our planned research. This is followed by a listing of the personnel on the project, a proposed budget and the current and pending federal support of the principal investigators.
\end{abstract}




\section{Introduction}

During the past few years the engineering of cobalt alloys on chromium thin film media, in combination with the development of thin film magnetic head technology, have caused a major revolution in the storage density and cost of magnetic hard disk drive storage. For only a few hundred dollars one can now obtain a 200 megabyte drive, whereas only ten years ago one would have had to pay this amount for a 5 megabyte drive and the drive would have occupied 5 times the volume. These vast improvements in storage performance can be directly correlated to: the smoothness of the new thin film media allowing the magnetic recording head media spacing to be decreased, increases in the coercivity of the media allowing the magnetization bit pattern to be more closely packed, and decreasing media noise allowing greater track density. These engineered improvements in magnetic media performance have only recently begun to be understood and related to the microstructure of, and processing conditions used in making, the thin films. Nevertheless, based upon some understanding and considerable empirical evidence, various groups have recently begun to project the magnetic media requirements necessary to increase recording densities by two orders of magnitude to 10 gigabits per square inch [1].

The future requirements for magnetic recording media are formidable. The projections, based upon recording density and simple signal to noise models, are that magnetic domain sizes, and perhaps crystallographic grain sizes, must be reduced to about $15 \mathrm{~nm}$ from the current $40 \mathrm{~nm}$ size while the coercivity must be increased to 2500 to 4500 Oe from the current day values of around 1500 Oe. The domains must be extremely well isolated from one another in order to achieve the low noise requirement and, at the same time, they must also be magnetically dense in order to provide a high readback signal level. The high anisotropy value of cobalt would imply that these 
characteristics might be achieved by current alloys. However, practically speaking, today's coercivities are considerably below the theoretical limit. This is principally because an incomplete understanding of the microstructural issues of anisotropy, orientation, the role of grain size, phase segregation, crystallographic faults, and interface phenomena and how these properties are related to the film preparation process, currently limit one's ability to manipulate the process, and hence the properties.

In the work performed over the past two years we have focussed on the structural aspects of magnetic thin films. We have investigated the microstructure and nanostructure of magnetic thin films and related them to their magnetic properties. In our proposed work we plan to continue this fundamental approach it understanding the role of structure on the magnetic properties. Our goal is scientific in nature, namely to understand how magnetic properties are affected by the structure of the materials. Our findings, however, should be of help in the development of materials with ever better magnetic properties for recording media, permanent magnets, and magnetic field sensors. 


\section{Review of Results to Date (August 1990 - October 1992)}

We have pursued two lines of research during the first two years of this work. In the main portion of the project we have performed fundamental investigations of the microstructure and crystallographic texture of $\mathrm{Co}$ based alloys deposited on $\mathrm{Cr}$ underlayers. This has included atomic resolution electron microscopy of the crosssections of $\mathrm{CoNiCr}$ on $\mathrm{Cr}$ thin films, studies of the microstructures and properties of magnetic thin films produced on single crystal $\mathrm{Cr}$ underlayers as well as magnetic films with interlayers of $\mathrm{Cr}$, and studies of the development of the crystallographic texture of $\mathrm{Cr}$ thin films. The other portion of our work has centered on understanding the crystallography and crystal structure of $\mathrm{SmCo} / \mathrm{Cr}$ thin films. These $\mathrm{SmCo} / \mathrm{Cr}$ films have the potential of being utilized as high density media, but their structure is not yet understood.

\section{Atomic Resolution TEM of CoNiCr Films}

During the initial portion of this program we performed atomic resolution studies of the interface between the $\mathrm{Cr}$ underlayer and $\mathrm{CoNiCr}$ thin films (see references [2] and [3]). This enabled us to determine that the $\mathrm{Cr}$ underlayer was crystalline right up to its interface with the Co-based alloy. See Figure 1. Various orientation relationships were observed directly, as were defects in the crystalline structure. These atomic level defects were most often stacking faults, and extended through the entire thickness of the Cobased (hcp) grains.

These faults form during the deposition process. The number of faults increases with deposition temperature, probably due to the lowering of the stacking fault energy, due to the increasing stability of the fcc phase with respect to the hep phase. We believe these faults are crucial for the development of high coercivity. One explanation for the importance of the faults is that $\mathrm{Ta}$ or $\mathrm{Cr}$ segregate to the faults (Suzuki effect) causing impediment of domain wall motion. Controlled experiments varying the number of 


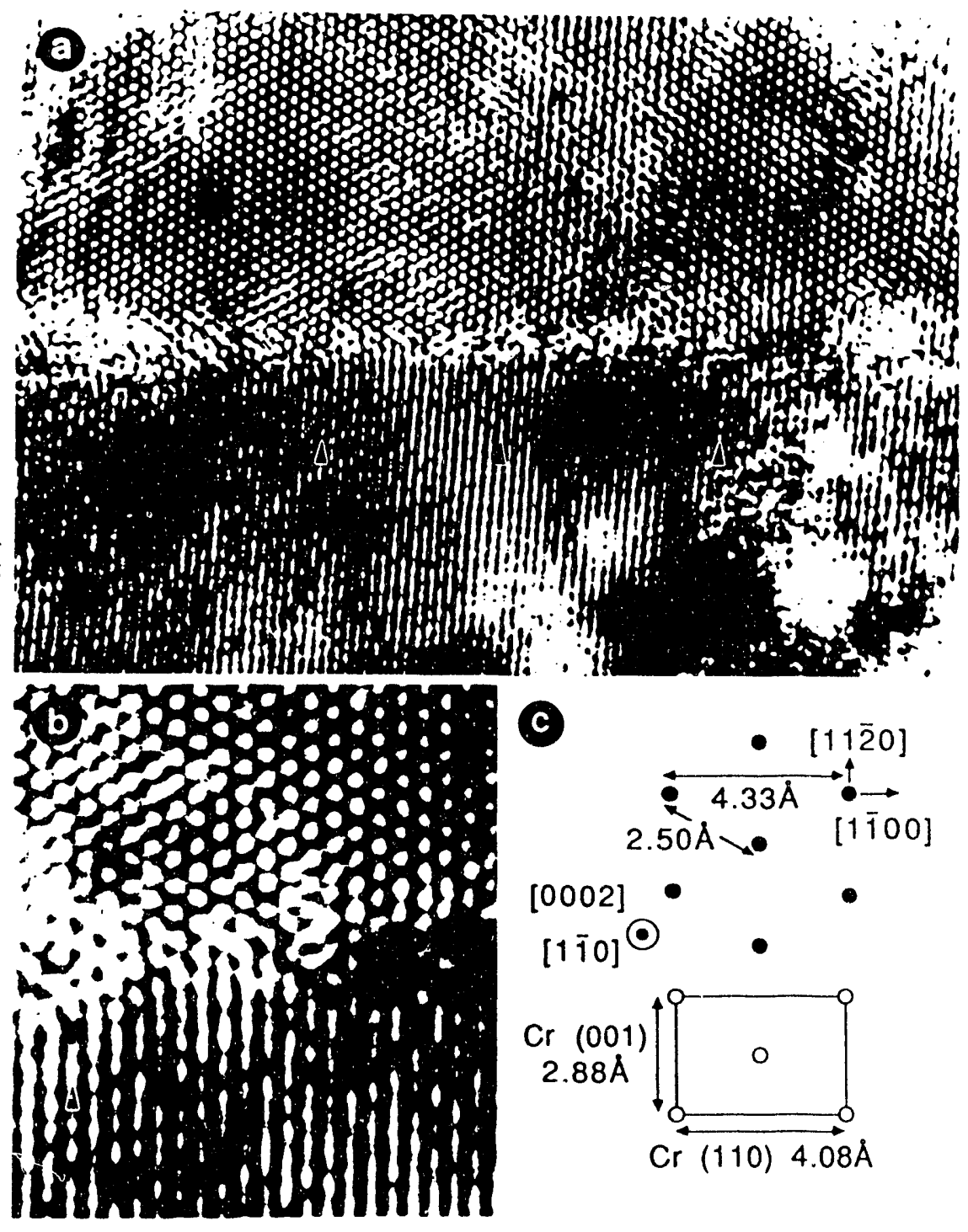

Figure 1. High Resolution Transmission Electron Microscopy image of the interface between $\mathrm{CoNiCr}$ and $\mathrm{Cr}$. From the image and the accompanying schematic the orientation relationship can be seen to be the Pitsch-Schrader. 
defects per unit volume, holding other parameters fixed, will be performed. Also, we propose to study the effect of stacking faults on coercivity in pure Co films. This will help us to distinguish the effect of structural faults from that of chemical segregation.

\section{CoNiCr and CoCrTa Thin Films}

In our initial studies we investigated the properties of thin films deposited onto single crystal substrates. See reference 4 . Among the interesting findings was that two variants of the hcp Co based alloy formed on the (100) Cr single crystal, with their c axes parallel to [110] and [1 $\overline{1} 0]$ of the Cr single crystal. See Figure 2. These variants had an effective grain size which seemed to vary with the processing conditions. The formation of such a two variant microstructure demonstrates that by careful control of the underlayer, very specific crystallographic textures can be produced in the magnetic layers.

More recently we have been studying double layer magnetic films, which have thin nonmagnetic isolation layers, or interlayers. Such films have been reported to have both low noise and high signal to noise ratios [5,6]. A large amount of work has been done to investigate the effect of the interlayer from the point of view of magnetic interaction. However, all the explanations to date have been phenomenological in nature. Furthermore it has been assumed that each magnetic layer was identical in microstructure. No studies on the microstructures of the multilayer magnetic thin films had been reported prior to our studies. We have investigated the role of microstructure in multilayered CoCrTa thin films. Our goal was to understand the relationship between various sputtering processes and film microstructures and the relationship between these microstructures and the magnetic properties.

Our initial findings were published in reference [7]. We showed that the second layer of the Co-based alloy had the same crystallographic texture as the first layer, for

films sputtered at room temperature. This implied that the $\mathrm{Cr}$ interlayer also had the 
same crystallographic texture as the $\mathrm{Cr}$ underlayer and that it acted as a template for the second Co-based alloy layer. Later cross sectional ARTEM work verified this mechanism of texture build up [8].

\section{Development of Texture}

During our attempt to control the crystallographic texture of the magnetic thin films, it became clear that we should investigate in more detail the mechanism of formation of the crystallographic textures. Since the texture of the Co-based alloys depends on that of the $\mathrm{Cr}$ underlayer, we first sought to understand what processes control the development of the $\mathrm{Cr}$ texture.

We produced $\mathrm{Cr}$ films with systematic variations of RF sputtering conditions such as substrate bias, sputtering power and Ar pressure. We found that we could control the texture of the $\mathrm{Cr}$ films by these variables just as we could by substrate temperature. We are currently developing a model for the way that the texture forms based on the mobility of adatoms and the kinetic energy of the bombarding particles. Another important variable to consider in the formation of texture is the relative surface energies of the free $\mathrm{Cr}$ surface $\{100\}$ or $\{110\}$ and the $\mathrm{Cr} /$ substrate interface energy. If the latter is high, the developing $\mathrm{Cr}$ grains will be smaller, and the minimization of the $\{110\}$ surface area will be favored. As shown in Figure 3, this could favor $\mathrm{Cr}$ grains with (001) in-plane orientation. These results are currently being written up for publication [9]. 


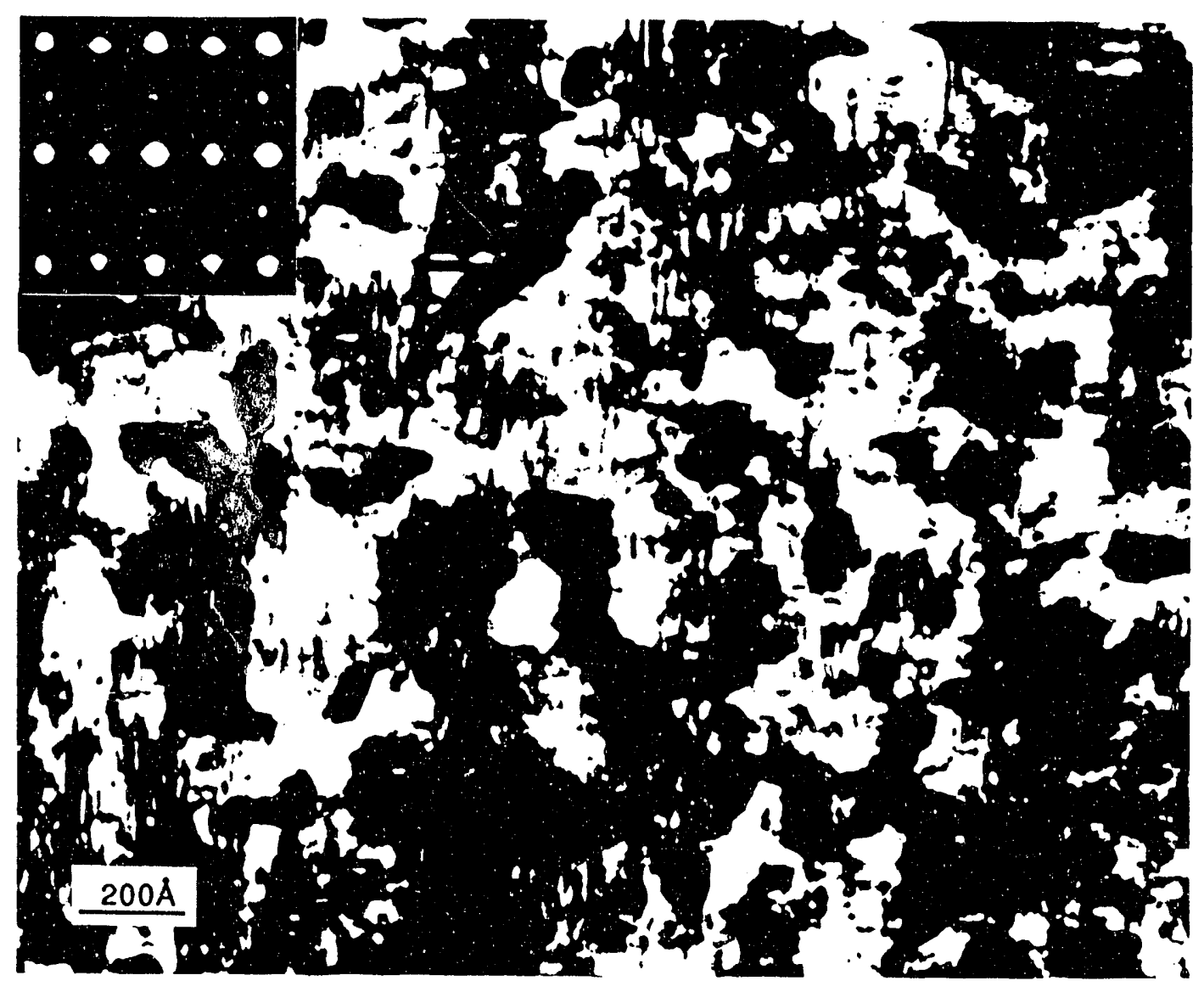

Figure 2. Bright field TEM of $\mathrm{CoNiCr} /(100) \mathrm{Cr}$, showing two hcp variants. Each variant has stacking faults along traces of the basal planes. 


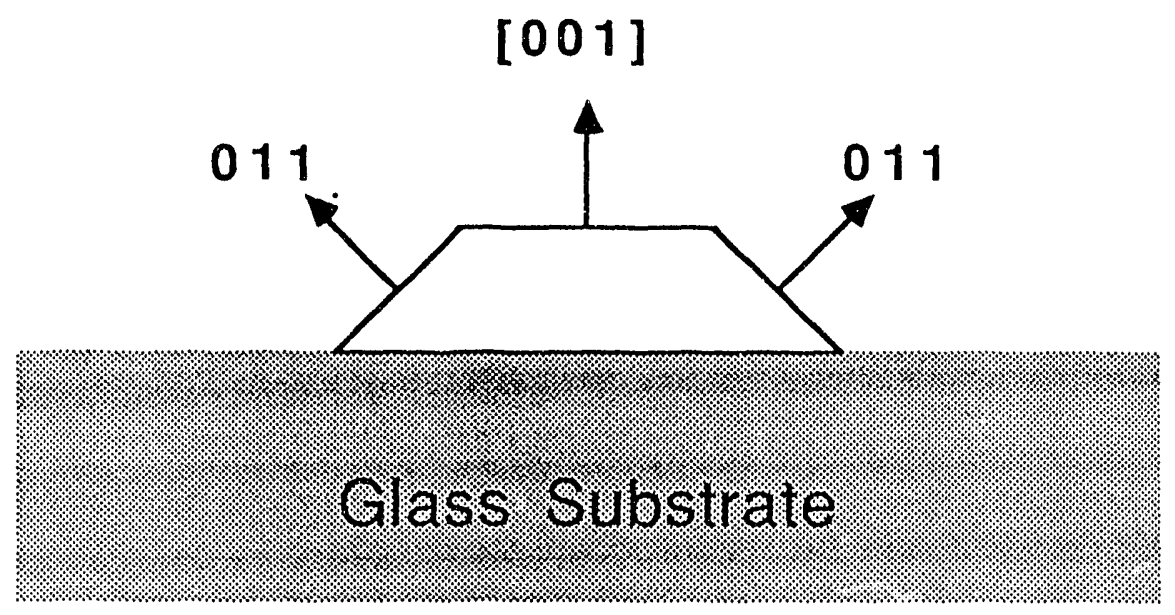

Figure 3. Schematic diagram of the formation of a $\mathrm{Cr}$ grain on a glass substrate. In this case the glass-Cr interfacial energy is large. Thus the shape of the $\mathrm{Cr}$ grain which minimizes its overall energy is one with (001) parallel to the substrate surface.

\begin{tabular}{|c|}
\hline Co-based (2) \\
\hline Cr Interlayer $\quad(1)$ \\
\hline Co-based (1) \\
\hline $\mathrm{Cr}$ Underlayer $\quad(\mathrm{U})$ \\
\hline
\end{tabular}

Figure 4. Schematic drawing of two Co-based films with a chromium underlayer (U) and a $\mathrm{Cr}$ interlayer (I). 


\section{CoSm/Cr Thin Films}

Current research activities in high density longitudinal magnetic recording media are concerned with increasing coercivity while reducing medium noise and transition width. To lower the medium noise it is necessary to obtain thin films with magnetically non-exchange coupled grains a few $\mathrm{nm}$ in size. To minimize the transition width a thin medium possessing a high coercivity is essential. To date we have obtained $\mathrm{SmCo} / \mathrm{Cr}$ thin films with in plane coercivities approaching 3000 Oe $[10,11]$. This portion of our research has involved investigating the microstructure and crystal structure of these SmCo thin films. The nanocrystalline grains have been seen to be smaller than $10 \mathrm{~nm}$. It has not been possible yet to identify the phases present but we believe both $\mathrm{Co}_{5} \mathrm{Sm}$ and $\mathrm{Co}_{17} \mathrm{Sm}_{2}$ may be present. Cross sectional TEM work continues in our attempt to characterize the structure of the films.

\section{Proposed Research}

Our studies so far have demonstrated that the extrinsic magnetic properties of thin films can be manipulated by varying the processing parameters used during their formation. Furthermore, we have investigated the way that the processing parameters control the properties, namely via the microstructure of this film. Nonetheless, much still needs to be done to understand more fully the role of specific microstructural features such as defects, crystallographic texture and chemical segregation. Our proposed work centers on these aspects.

\section{Interlayers}

The study of how interlayers can be used to manipulate the properties of thin films will be a major segment of the research program over the next several years. We seek to develop ways of systematically varying the microstructure of the two Co-based films. For example, in Fig. 4 we see that there are two Co-based films (labeled 1 and 2) and two $\mathrm{Cr}$ layers, labeled $\mathrm{U}$ and $\mathrm{I}$, for underlayer and interlayer. The cryst: 1 lographiz 
texture of the second $\mathrm{Co}$ film depends on that of the $\mathrm{Cr}$ interlayer. If the $\mathrm{Cr}$ in:erlayer has the same texture as the $\mathrm{Cr}$ underlayer, the two Co-based films will have the same texture. Se: for example reference [7]. If, however, we make the crystallographic texture of the $\mathrm{Cr}$ interlayer different from that of the underlayer, we could obtain Co-based layers with different texture. This will allow us to directly obtain information about the way that texture controls magnetic properties. It may be that the films produced with Co layers of differing crystallographic texture will have iuproved magitetic recording properties. To investigate this, we will produce films that have a systematic variation in texture and document how such variation affects the magnetic properties of the film.

We will also produce SmCo films with $\mathrm{Cr}$ interlayers. To date we have only investigated single layer films of $\mathrm{SmCo}$ on $\mathrm{Cr}$. However, if the nanocrystals of the $\mathrm{SmCo}$ alloy form mainly at the $\mathrm{SmCo} / \mathrm{Cr}$ interface, it may be best to produce films with several such interfaces, i.e., produce films that have one or two $\mathrm{Cr}$ interlayers.

\section{Texture Development and Effect on Coercivity}

\section{a. Cr Films}

We will continue our fundamental studies of the development of texture of the $\mathrm{Cr}$ underlayers. The ideas we have proposed for the mechanism of the development of $\{100\}$ texture must be more thoroughly investigated by varying the processing parameters in such a way as to either maximize or minimize the $\{100\}$ texture. If the mechanism of the formation of the $\{100\}$ texture can be fully understood, other ways of producing it should become clear.

We will also study how the $\{100\}$ texture of the $\mathrm{Cr}$ films switches to that of $\{110\}$ as film thickness is increased. Although this feature has been documented $[12,13]$ the crystallographic mechanisms have not been documented. If these mechanisms can be documented, it is believed that a better control of the textures will be possible. Better 
control of film texture will allow greater latitude in process conditions so that the microstructure and hence magnetic properties can be manipulated to a greater degree.

\section{b. Co films}

The development of the crystallographic texture of Co-based magnetic thin films on $\mathrm{Cr}$ underlayers has been thoroughly documented [2] and explained in terms of epitaxial growth. However, the effect of the crystallographic texture on magnetic properties has been less well understood.

One of the difficulties of obtaining a correlation between the crystallographic texture of the Co-based film and in-plane coercivity is that when films with different crystallographic textures are produced, various other processing parameters and henc microstructural features are varied. For example, in order to produce Co-based films with the $\mathrm{c}$ axes in the film plane, $\{100\} \mathrm{Cr}$ underlayers are usually used. These are usually obtained at elevated deposition temperatures $\left(\sim 260^{\circ} \mathrm{C}\right)$. Thus, to produce Co-based films with their $\mathrm{c}$ axes in plane, the temperature of deposition must be high. This, however changes the grain size and the amount of chemical segregation which occurs within the Co-based films. Indeed, such segregation is thought to have large effects on the coercivity.

In order to eliminate the effects of chemical segregation with the films on coercivity, we will make pure Co films with different crystallographic texture. This will produce films with lower coercivities, but since our goal is to isolate the effect of crystallographic texture on the coercivity, this does not matter. By using pure Co films we will also have a better knowledge of such parameters as the hcp/fcc transition temperature and the stacking fault energy since these values are known for pure Co. This will enable us to explain the changes in the number of defects with deposition temperature, and thus io Better control the microstructure of the magnetic films.

Some work has been performed on pure Co films $[14,15]$. However the focus of the previous work was how the properties varied with deposition temperature, not how 
they varied with the structural defects of the thin film. In our proposed work we will not only vary the deposition temperature, but also determine how this affects the microstructure of the film. It is the structure vs. property aspect that will be the focus of our studies.

\section{SmCo Structure}

As indicated in our summary of work to date, we believe we have found that nanocrystals exist in the thin films of SmCo that are deposited on $\{110\}$ textured $\mathrm{Cr}$ underlayers. We believe that this is critical for the high in plane coercivity of this alloy $(\sim 3000 \mathrm{Oe})$. However, when SmCo is deposited on (100) textured $\mathrm{Cr}$ underlayers the in plane coercivity is drastically reduced $(-900 \mathrm{Oe})$. Also, when $\mathrm{SmCo}$ is deposited directly onto a glass substrate, without the $\mathrm{Cr}$ underlayer, the in-plane coercivity is low $(\sim 700$ Oe). Furthermore, when deposited without the $\mathrm{Cr}$ underlayer, the magnetic recording noise performance has been found to be inferior to the films prepared on $\mathrm{Cr}\{110\}$ textured underlayers. The structural reasons for this are proposed to be investigated by means of atomic resolution TEM.

We plan to carefully docurnent the structures of the SmCo films deposited on these various underlayers by means of atomic resolution TEM. In particular, for the high in plane coercivity SmCo films we will determine if the structure consists of only nanocrystals at the $\mathrm{Cr}$ interface, or if it consists of a mixture of nanocrystals in a matrix of amorphous material. Also, we will try to determine which of the SmCo crystalline phases are present in the films and how the phases may vary across the film thickness. The films with lower in plane coercivity will also be examined by atomic resolution TEM to determine how their structures differ from that of the high in plane coercivity films. We will determine if nanocrystals are present, and if so whether they are arranged differently in the structure (i.e., less crystallographic texture, more amorphous phase, etc.). These results will enable us to directly correlate the structure of the SmCo films with their magnetic propertics. 


\section{IV: Summary}

In this proposal we presented our plans to continue our research program on the role of microstructure on magnetic properties of thin film materials. In the first section we summarized our results to date. We have pursued our plans to document how the microstructure of thin films control their magnetic properties. Our high resolution electron microscopy results demonstrated that defects in Co-based films may be responsible for higher coercivity. We have not yet been able to document whether the faults themselves act to increase the coercivity, or if chemical segregation to the faults enhances their potency. To resolve this we propose to study pure Co films and document how the defect density affects coercivity. This will allow us to isolate the structural effects from the chemical segregation effects.

We also presented our findings on the effects of $\mathrm{Cr}$ interlayers on the microstructure of the second $\mathrm{Co}$-based film in $\mathrm{Co} / \mathrm{Cr} / \mathrm{Co} / \mathrm{Cr}$ multilayer films. Our proposed work will further explore the role of interlayers in controlling the structure of films. In particular, we will vary the crystallographic texture of the interlayer in an attempt to magnetically decouple the two Co-based films from each other in these multilayer films. Furthermore, we plan to continue our studies on the development of the Cr underlayer texture.

Finally our work on $\mathrm{SmCo} / \mathrm{Cr}$ films was presented. The future work in this area lies in the complete documentation of the microstructure of these films, including the identification of the nanocrystalline phase(s) and amorphous phase (if any) present in the films with very high ( $3000 \mathrm{Oe})$ and much lower coercivity ( $700 \mathrm{Oe})$. Understanding the structure here will help us to further the role of very small crystals in controlling in plane coercivity.

Our laboratory facilities allow us to produce our own films and to characterize them magnetically. Furthermore, our electron microscopy facilities allow us to show how changing the processing of films effects the magnetic properties through the variation in microstructure. This fully integrated research program thus allows us to directly vary the major parameters that control thin film magnetic properties, and hence to ascertain their individual contributions to the changes in properties which they produce. 


\section{References}

1. Edward S. Murdock, "Roadmap for $10 \mathrm{Gbiv/n^{2 }}$ Media: Challenges," IEEE Trans. Magn. 28(5), 3078 (1992).

2. D. E. Laughlin and B. Y. Wong, "The crystallography and texture of Co-based thin film deposited on Cr underlayers," IEEE Trans. Mag. 27(6), 4713 (1991).

3. B. Y. Wong and D. E. Laughlin, "A high-resolution TEM investigation of the interfacial structure of $\mathrm{CoNiCr} / \mathrm{Cr}$ bi-layer magnetic thin films," Proceedings of 49th Annual Meeting of Electron Microscopy Society of Ainerica, San Francisco Press, San Francisco, CA (1991).

4. B. K. Wong, D. E. Laughlin and D. N. Lambeth, "Investigation of CoNiCr thin films deposited on (100) and (110) Cr single crystals," IEEE Trans. Magn. 27(6), 4733 (1991).

5. E. S. Murdock. B. R. Natarajan, R. G. Walmsley, "Noise properties of multilayered Co-alloy magnetic recording media," IEEE Trans. Magn. 26, 2700 (1990)

6. S. E. Lambert, J. K. Howard and I. L. Sanders, "Reduction of media noise in thin film metal media by lamination," IEEE Trans. Magn. 26, 2706 (1990).

7. Y. C. Feng, D. E. Laughlin and D. N. Lambeth, "Texture of $\mathrm{Cr}$ interlayer in double $\mathrm{CoCrTa}$ thin films and effects of interlayer on the magnetic properties," IEEE Trans. Magn. 28(5), 3270 (1992).

8. B. Y. Wong and D. E. Laughlin, "The cross sectional structures of $\mathrm{CoNiCr} / \mathrm{Cr}$ bilayer and multilayer thin films," accepted for publication, Applied Physics Letters.

9. Y. C. Feng, D. E. Laughlin and D. N. Lambeth, "The formation of crystallographic texture in RF sputter deposited $\mathrm{Cr}$ thin films," in preparation.

10. E.M.T. Velu and D. N. Lambeth, "CoSm-based high coercivity thin films for longitudinal Recording," J. Appl. Phys. 69(8), 5175-5177, 1991.

11. E.M.T. Velu and D.N. Lambeth, "High Density Recording on SmCo/Cr Thin Film Media," IEEE Trans. on Mag. 28(5), 3249-3254 (1992).

12. S. L. Duan, J. O. Artman, B. Wong and D. E. Laughlin, "Study of the growth characteristics of sputtered Cr thin films," J. Applied Phys. 67(9), 4913 (1990).

13. S. L. Duan, J. O. Artman, B. Wong and D. E. Laughlin, "The dependence of the microstructure and magnetic properties of $\mathrm{CoNiCr} / \mathrm{Cr}$ thin films on the substrate temperature, IEEE Trans. Magn. 28(5), 1587 (1992).

14. J. Daval and D. Randet, "Electron microscopy on high coervive force $\mathrm{Co} / \mathrm{Cr}$ composite Films," IEEE Trans. Magn. 6(4), 768 (1970).

15. T. Yeh, J.M. Sivertson and J.H. Judy, "Magnetic properties and micromagnetic character of RF sputtered Co thin films on thin Cr underlayers," pp. 15-20, published in Magnetic Materials: Microstructure and Properties, edited by $\mathrm{T}$. Suzuki, Y. Sugita, B. Clemens, K. Ouchi and D. Laughlin, MRS (1991). 


\section{Publications and Presentations}

\section{Publications}

B.Y. Wong, D.E. Laughlin and D.N. Lambeth, "Investigation of CoNiCr thin films deposited on [100] and [110] Cr single crystals," IEEE Trans. Mag. 27(6), 4733-4735 (1991)

D.E. Laughlin and B.Y. Wong, "The crystallography and texture of Co-based thin film deposited on Cr underlayers," IEEE Trans. Mag. 27(6), 4713-4717 (1991).

B.Y. Wong and D.E. Laughlin, "A high-resolution TEM investigation of the interfacial structure of $\mathrm{CoNiCr} / \mathrm{Cr}$ bi-layer magnetic thin films," Proceedings of 49 th Annual Meeting of Electron Microscopy Society of America (EMSA), San Francisco Press, San Francisco, $\therefore$ A, 1991.

E.M.T. Velu and D. N. Lambeth, "CoSm-based high coercivity thin films for longitudinal Recording," J. Appl. Phys. 69(8), 5175-5177, 1991.

E.M.T. Velu and D.N. Lambeth, "High Density Recording on $\mathrm{SmCo} / \mathrm{Cr}$ Thin Film Media," IEEE Trans. on Mag. 28(5), 3249-3254 (1992).

Y.C. Feng, D.E. Laughlin and D.N. Lambeth, "Texture of Cr Interlayers in Double CoCrTa Thin Films and Effects of Interlayers on their Magnetic Properties," IEEE Trans. on Mag. 28(5), 3270-3272 (1992).

\section{Papers in Preparation}

Y.C. Feng and D.E. Laughlin, "The Existence of the Co3Ta Phase in CoCrTa Targets," to be submitted to Scripta Metallurgica.

Y.C. Feng, D.E. Laughlin and D.N. Lambeth, "The Formation of Crystallographic Texture in RF Sputter Deposited Cr Thin Films," to be submitted to Journal of Applied Physics.

\section{Oral Presentations}

B.Y. Wong, D.E. Laughlin and D.N. Lambeth, "Investigation of CoNiCr Thin Films Deposited on [100] and [110] Cr Single Crystals," presented at IEEE Intermag, June, 1991.

D.E. Laughlin and B.Y. Wong, "The Crystallography and Texture of Co-Based Thin Films Deposted in Cr Underlayers," presented at IEEE/Intermag Conference, June, 1991.

B.Y. Wong and D.E. Laughlin, "A High Resolution Transmission Electron Microscopy Investigation of the Interfacial Structure of $\mathrm{CoNiCr} / \mathrm{Cr}$ Bi-layer Thin Films," presented at the 49th Annual Meeting of the Electron Microscopy Society of America, San Diego, CA, August, 1991. 
D.E. Laughlin, "Studies on Thin Film Magnetic Materials," NTT Labortories, Mito, Japan, March 18, 1992.

D.E. Laughlin, "Studies on $\mathrm{CoNiCr}$ and CoCrTa Thin Films," Hitachi Metals Ltd., Tsuchiura, Japan, March 19, 1992.

D.E. Laughlin, "High Resolution TEM Studies of Magnetic Thin Films," Hitachi Metals, Ltd., Kumagaya, Japan, March 20, 1992.

D.E. Laughlin, "High Resolution TEM Studies of Thin Film Magnetic Media" Tohoku University, Japan, March 23, 1992.

Y.C. Feng, D.E. Laughlin and D.N. Lambeth, "Texture of $\mathrm{Cr}$ Interlayers in Double CoCrTa Thin Films and Effects of Interlayers on the Magnetic Properties," presented at the 1992 INTERMAG Conference, St. Louis, MO, April, 1992.

E.M.T. Velu and D.N. Lambeth, "High Density Recording on SmCo/Cr Thin Film Media," presented at the 1992 INTERMAG Conference, St. Louis, MO, April, 1992. 


\section{RESEARCH FACILITIES}

The research facilities at $\mathrm{CMU}$ directly relevant to this research proposal are described briefly below. Each of the Central Research Facilities charge for usage of instruments at varying rates. These have been taken into account in the budget.

\section{Clean Room Facility}

In 1983 , at a cost of $\$ 1.6$ million, the University constructed a 4000 square foot clean room facility for the Magnetics Technology University. The facility uses vertical laminar air flow, with sidewall return air chases throughout. A 1500 square foot area, rated as class 20 , is used for the most critical tasks, such as mask making, wafer and substrate cleaning, photolithography and SEM inspection. A long $8 \mathrm{ft}$. wide class 100 corridor provides access to processing equipment in four class 100 bays, each measuring $12 \mathrm{ft}$. by $27 \mathrm{ft}$.

Various pieces of equipment which are used for the preparation, fabrication and characterization of data storage materials and devices, are housed in the facility. Five sputtering systems are available for the deposition of magnetic and magneto-optic materials. A Perkin-Elmer model $2400 \mathrm{~J} 6$ has three six-inch targets and operates in the RF-diode mode. A Perkin-Elmer model 2400 equipped with eight-inch targets is used in the RF-diode and DC-magnetron modes. An Anelva model SPF-730 has three eight-inch targets, RF diode sputters upward and is used for depositing magnetic media. A Leybold Heraeus Model Z400 has three three-inch targets and may be operated in either RF or DC diode or magnetron modes. It has a load lock so sample turn-around time is very short. The largest system, which is used extensively for sputtering magnetic and magnetooptical disks, is a Leybold Heraeus Model 650. It is an in-line system with a load lock and two deposition chambers. One chamber is used for the magnetic material deposition, and the other is used for the overcoat. The substrate table rotates under the eight-inch targets to improve uniformity of deposition. Like the smaller Z400, it can be operated either in RF or DC diode or magnetron modes.

In addition, the Clean Room houses a Commonwealth Scientific Dual Millatron Ion Beam Deposition System. An ultra-clean ion-pumped vacuum system with resistance and electron-beam heated sources is available for depositing contacts on semiconductor and magnetic devices. For growth of both magnetic and semiconductor materials, liquid phase and vapor phase reactors are available. 
The facility also has a small vacuum evaporator, a barrel piasma etcher,several furnaces, an ultrasonic wire bonder, and a $200 \mathrm{KeV}$ ion implanter.Recent additions include a plasma deposition system and a reactive ion etching system. Also in the clean room is equipment for fabrication and reproduction of photolithographic masks including a 100:1 reduction camera (4 $\mu \mathrm{m}$ resolution), a 10:1 reduction camera with $1 \mu \mathrm{m}$ resolution, a Cobilt mask aligner, and a deep-ultraviolet Karl Suess contact aligner with $0.5 \mu \mathrm{m}$ resolution and $0.1 \mu \mathrm{m}$ alignment accuracy. Apparatus for spinning, baking and developing photoresist is available, as is an oxygen plasma asher for removing photoresist residues.

Submicrometer line width masks, which may be reproduced on the deep-UV contact aligner, are fabricated at Cornell's National Nano Fabrication Facility, with mask layout done on Carnegie Mellon University computer-aided design (CAD) facilities. Materials and device inspection equipment, which are located in the clean room, include a Tencor Alpha Step 200 surface profilometer, a Hitachi Model S-800 field emission SEM, an optical microscope, and an interferometer for film thickness measurements. Adjacent to the clean room is a shop with machine tools and other repair/maintenance equipment.

\section{Magnetic Measurements Facility}

The University maintains a facility for measurements on magnetic materials and devices. A PAR vibrating sample magnetometer, a torque magnetometer, a magnetoresistance measurement system, a spectrum analyzer, and a SHB instruments high-sensitivity hysteresis-loop tracer are used for magnetic materials characterization. A SQUID magnetometer has recently been purchased with support provided by the National Science Foundation and the University. During 1992, a Digital Measurement Systems Vector vibrating sample magnetometer and a torque magnetometer were added.

In addition to commercially available characterization tools, the University has constructed various specialized and unique instruments. A high resolution $(0.3 \mu \mathrm{m})$ scanning magneto-optic photometer with $250 \mathrm{MHz}$ bandwidth is available for small spot Kerr magneto-optic measurements on magnetic materials and devices. This system has been used extensively for measurements on thin film, ferrite and MIG heads. Three magneto-optical testers employing Leitz Orthoplan polarized-light microscopes are used 
for domain imaging. A Perceptics Model 9200 image processing system is available for use with these systems. Two of these domain imaging systems provide 4 -nsec exposure time recordings of domain patterns in devices in operation at high frequency. One is set up for observations by the polar Kerr effect and one is set up for observations by the longitudinal Kerr effect. The system set up for the polar Kerr effect is used for measurements on magneto-optical recording materials, while the system set up for the longitudinal Kerr effect is used for dynamic measurements on magnetic recording heads.

There are also several other major measurement facilities. Magnetic disk test stands, fully equipped for spectrum analysis and waveform digitization have been donated by Sponsors and are used for measurements on rigid disks. One of the stands has been donated by sponsors and is capable of characterizing signal and noise as functions of recording density overwrite field, or erase field. Another, a Cambrian Super Spin Stand, is a highly automated research and engineering tool aimed at the development of $\mathrm{read} / \mathrm{write}$ heads, media, and interface electronics. An optical disk test stand with an air bearing spindle and an air bearing slider with a laser interferometer for positioning control is being set up. FMR spectrometers are available for characterization of materials. An X-band instrument employs a TE(011) circular cavity $(9.3 \mathrm{GHz})$. A microstrip spectrometer is operable from low frequency to $8.5 \mathrm{GHz}$, and a $35 \mathrm{GHz}$ computer controlled spectrometer incorporates a $30 \mathrm{kG}$ magnet. Equipment is available for concurrent microwave excitation and optical observation of domains. In addition, with funding from the Air Force Office of Scientific Research and the University, we have added a spin polarized electron microscopy facility at a total cost of about $\$ 1,000,000$. (See Electron Microscopy section below.)

The University also maintains facilities to disperse, characterize, and coat particulate magnetic media. Red Devil vibratory shakers and $250 \mathrm{ml}$ Eiger Ball Mills are used for dispersing magnetic particulate. A 45 degree Glossmeter, a Steinberg Associates Dispersion Quality Monitor (DQM),viscometers and a Rheological Magnetic Analyzer are available for characterizing dispersion quality. Coating equipment includes an Erichssen 8.5 inch x 15 inch mechanical-draw knife-edge coater, a hand-draw coater,and a spin coater. In addition, the University is in the process of acquiring a bench-top roll coating facility that will enable long lengths of media to be coated. 


\section{Electron Microscopy}

The Electron Microscopy Facility contains four transmission electron microscopes (JEOL JEM 120CX TEMSCAN, Philips 420T 'TEM/STEM, JEOL 4000-EX and a Vacuum Generator HB501 HV STEM), two scanning electron microscopes (CamScan Series 4 and JEOL JSM 35), as well as peripheral supporting equipment.

The JEOL 120CX and Philips 420T transmission electron microscopes (TEM) are equipped for scanning transmission electron microscopy (STEM) and also have microdiffraction capabilities. For energy dispersive analysis, both instruments have fully quantitative PGT System 4 plus series energy-dispersive analyzer systems with imaging which provide local chemical analysis capabilities for regions as small as $25 \mathrm{~nm}$. The Philips also is equipped with a Gatan parallel Electron Energy Loss Spectrometer (EELS).

The JEOL $4000 E X$ is an ultra high resolution TEM (1.7 $\AA$ point-to-point) with a double tilt hot stage $\left(800^{\circ} \mathrm{C}, 1.8 \AA\right.$ resolution) and a Gatan Image Intensifier TV system. The HB501 STEM is an ultra high vacuum field emission microscope with a beam size as small as $5 \AA$. This microscope will have a spin polarization analyzer attached to be used in the scanning mode (SEMPA - Scanning Electron Microscope for Polarization Analysis).

Both scanning electron microscopes (SEM) are also equipped with energy dispersive $x$-ray analyzers for fully quantitative elemental chemical analysis. The CamScan Series 4 has a PGT System IV analyzer, while the JEOL JSM 35 has a PGT System IV. The CamScan Series SEM is a state-of-the-art instrument with a distortion free, full TV rate scan generation system. A motor-driven eucentric stage provides working distances from 10 to $100 \mathrm{~nm}$. Capabilities include advanced video and scan processing for secondary electron imaging, back scattered electron imaging, and absorbed current imaging.

Supporting equipment includes several wafering machines, thin-foil preparation equipment, three ion milling machines, two vacuum evaporators, an RF sputterer for specimen preparation, and darkroom facilities for photographic processing. A full-time engineer/microscopist is assigned to this facility for training, routine maintenance, and general supervision.

\section{Surface \& Micro Analysis}

The Surface \& Micro Analysis facility provides capabilities for Auger electron spectroscop $y$ (AES), $x$-ray photoelectron spectroscopy (XPS), and low energy electron diffraction (LEED). 
In one instrument, the Multi-Purpose Surface Analyzer (an AES, XPS, and LEED system), a coaxially mounted electron gun serves as the source for Auger studies and a double pass cylindrical mirror analyzer provides the necessary high-resolution capabilities. For XPS studies, a dual-anode $\mathrm{x}$-ray source capable of providing both $\mathrm{Mg} \mathrm{K}$ alpha and $\mathrm{Al} \mathrm{K}$ alpha soft $\mathrm{x}$-rays is available. An ion sputtering system, which has an ion gun capable of high sputtering rates and ion beam rastering, enables simultaneous depth profiling of several elements. A quadrupole mass spectrometer (UTI Model 100C) enables gas constituents to be measured and monitored in this system.

Another instrument, a Scanning Auger Microprobe (Physical Electronics Model 600 ), provides the most sophisticated Auger instrumentation available. This completely antomated instrument has a nominal resolution of $35 \mathrm{~nm}$. Auger spectra can be acquired in a number of different modes including point analysis, depth profiling of several elements simultaneously, and mapping of microstructures.

Auger electron spectroscopy capabilities are also available in a system being used primarily for the study of catalytic materials and surface reactions on solids. This system also has a quadrupole mass spectrometer and a sample carousel with heating and cooling capabilities. The system design allows for the addition of LEED, sputtering and other options.

\section{X-Ray Analysis Facility}

The X-Ray Analysis Facility has nine $x$-ray generators (three Siemens Kristalloflex 4, two Siemens Kristalloflex 2, two Rigaku, one Philips, and an Elliot rotating anode), three horizontal diffractometers (two Siemens and one Rigaku) employing Bragg-Brentano geometry, a vertical diffractometer (Philips) employing the same tyje of geometry, a Guinier Thin Film Glancing-Angle goniometer (Huber) mploying Seemann-Bohlin geometry, a pole figure goniometer (Siemens), a pole figure atiachment to the Rigaku diffractometer, and a fluorescence spectrometer (Siemens). The facility also has a number of Debye-Scherrer, Laue back-reflection, Precession, and various other $x$-ray cameras. Peripheral supporting equipment includes incident and diffracted beam monochromators, low and high temperature attachments to the Rigaku diffractometer, densitometers, view boxes, and the powder diffraction files on CD ROM. 


\section{Personnel}

During the Fall 1990, Prof. Lambeth had one of his post-doctoral associates (Dr. E.M.T. Velu) spend a portion of his time on the project. Prof. Laughlin had one of his more senior graduate students (Mr. B. Wong) pursue atomic resolution electron microscopy of the thin films.

We now have one graduate student currently supported on the project, Mr. Y.C. Feng from the People's Republic of China. Mr. Feng was accepted as a student in the Materials Science and Engineering Department in January of 1991 and passed his Ph.D. qualifying exam earlier this year. He has expertise in electron microscopy and magnetic materials.

The resumés of the two principal investigators follow. 


\section{Dạvid E. Laughlin}

Professor of Materials Science Engincering

Carnegie-Mellon University

Pittsburgh, PA 15213-3890

2357 McNary Boulevard

Pittsburgh, PA 15235

(412) 268-2706

(412) 244-1950

\section{Education:}

Ph.D. Metallurgy and Materials Science, 1973

Massachusetts Institute of Technology

B.S. Metallurgical Engineering, 1969

Drexel University

Professional Experience:

Carnegie-Mellon University:

1982 - present
1986 - 1989
1988 - present
$1982-1988$
$1979-1982$
$1974-1979$
$1973-1974$
$1969-1971$
$1971-1973$
1970

1982 - present

$1986-1989$

$1982-1988$

$1979-1982$

$1974-1979$

$1973-1974$

$1971-1973$

1970

\author{
Professor \\ Director, Magnetic Materials Research Group \\ Editor, Metallurgical Transactions \\ Associate Editor, Metallurgical Transactions \\ Associate Professor \\ Assistant Professor \\ NAS-NAE Post-Doctoral Associate, National Bureau of Standards \\ Teaching Associate, Massachusetts Institute of Technology \\ Research Associate, Massachusetts Institute of Technology \\ Electron Microscopy Technician, Kennecott Copper
}

\section{Honors}

Fellow, ASM International

\section{Research Interests}

The research interests of David E. Laughlin have centered around the investigation of phase transformations and structure by means of transmission electron microscopy. He has studied spinodal decomposition and ordering processes in several aluminum, copper and nickel based alloys as well as in III-V compounds by detailed analysis of their microstructure as well as electron diffraction patterns. Other fields in which he has been actively involved include the investigation and characterization of dislocation structure of twist boundaries between dissimilar materials, cellular precipitation and twinning of ordered materials. Currently he is studying ordering reactions in Fe-Pd alloys, and investigating the magnetic structure of hard magnetic materials as well as thin film magnetic recording media by Lorentz electron microscopy as well as atomic resolution microscopy. He is the director of both the X-ray Central Facility and the Electron Optics Central Facility of the Materials Science and Engineering Department of Carnegie Mellon University. He also is editor of the Metallurgical Transactions. Knowledgeable in a wide variety of X-ray and electron optical techniques, he has more than 140 technical publications in the field of phase tran sformations, physical metallurgy and magnetic materials, and has coedited the books Solid-Solid Phase Transformations and Magnetic Materials: Microstructure and Properties. 


\section{PUBLICATIONS}

\section{BOOKS AND EDITED VOLUMES}

Magnetic Materials: Microstructure and Properties, edited by T. Suzuki, Y. Sugita, B. Clemens, K. Ouchi and D.E. Laughlin, Materials Research Society, Pittsburgh, PA, 1991.

Solid-Solid Phase Transformations, edited by H.I. Aaronson, D.E. Laughlin, R.F.

Sekerka and C.M. Wayman, The Metallurgical Society of AIME, Warrendale, PA, 1982.

\section{RECENT JOURNAL ARTICLES}

H.S. Lessure, E.B. Boltich, S.G. Sankar, W.E. Wallace and D.E. Laughlin, "Anisotropic Paramagnetic Susceptibility of $\mathrm{RBa}_{2} \mathrm{Cu}_{3} \mathrm{O}_{7-x}$ Due to the Crystalline Electric Field: Point Charge Calculations", Journal of Less-Common Metals, 149, 441-446, 1989.

K. Hono, B.G. Demczyk and D.E. Laughlin, "Electron microdiffraction of faulted regions in Co-Cr and Co-Ni-Cr thin films", Appl. Phys. Lett., 55(3), 229-231, 1989.

K. Hono and D.E. Laughlin, "Evidence of Phosphorous Segregation in Grain'Boundaries in Electroless-Plated Co-P Thin Film", J. Mag. Mag. Matls. 80, L137-L141, 198?.

J.-W. Lee, M. Migliuoio, A.K. Stamper, D.W. Greve, D.E. Laughlin and T.E. Schlesinger, "Microstructure of superconducting $\mathrm{YBa}_{2} \mathrm{Cu}_{3} \mathrm{O}_{7-\delta}$ thin films on $\mathrm{Si}$ and alumina substrates with buffer layers," J. Appl. Phys. 66(10), 4886-4890 (1989).

S. Mahajan, M.A. Shahid and D.E. Laughlin, "Current status of atomic ordering and phase separation in ternary and quaternary III-V compound serniconductors," Inst. Phys. Conf. Ser. No. 100: Section 3, presented at Microsc. Semicond. Mater. Conf., Oxford, IOP Publishing, 143-153 (1989).

T.L. McDevitt, F.S. Turco, M.C. Tamargo, S. Mahajan, D.E. Laughlin, V.G. Keramidas and W.A. Bonner, "Effects of substrate orientation on phase separation in InGaAs and InGaAsP epitaxial layers," Inst. Phys. Conf. Ser. No. 100: Section 3, presented at Microsc. Semicond. Mater. Conf., Oxford, IOP Publishing, 173-180 (1989).

W.A. Soffa and D.E. Laughlin, "Decomposition and Ordering Processes Involving Thermodynamically First-Order Order $\rightarrow$ Disorder Transformations," Acta Met. 37, 30193029 (1989).

J.W. Lee, S.C.N. Cheng, M.H. Kryder and D.E. Laughlin, "The relationship between deposition conditions, microstructure and properties of RE-TM thin films," Materials Research Society Symposium Proc., 150, 159-164 (1989).

S.L. Duan, J.O. Aru :an, J.W. Lee, B. Wong and D.E. Laughlin, "Effect of sputtering conditions, annealing and the microstructure of $\mathrm{Cr}$ underlayer on the magnetic properties of $\mathrm{CoNiCr} / \mathrm{Cr}$ thin films," IEEE Trans. on Magnetics 25(5), 3884-3886 (1989).

J-W. Lee, D.E. Laughlin and S. Nam, "Direct observation of discommensurate walls in Bi-Ca-Sr-Cu-O superconductors," Phys. Rev. B 41(7), 4093-4097, 1990. 
S.F. Cheng, B.G. Demczyk, D.E. Laughlin and W.E. Wallace, "Phase analysis and microstructural characterization of $\mathrm{SmTiFe}_{11-\mathrm{x}} \mathrm{Co}_{\mathrm{x}}(\mathrm{x}=0,8,11)$ and $\mathrm{DyTiCo}_{1}$ by transmission electron microscopy," J. Mag. Mag. Mat. 84, 162-174, 1990.

S.L. Duan, J.O. Artman, B. Wong and D.E. Laughlin, "Study of the growth characteristics of sputtered Cr thin films," J. Appl. Phys. 67(9), 4913-4915, 1990.

S.L. Duan, J.O. Artman, K. Hono and D.E. Laughlin, "Improvement of the magnetic properties of CoNiCr thin films by annealing," J. Appl. Phys. 67(9), 4704-4706, 1990.

Y. Shen and D.E. Laughlin, "A transmission electron microscopy study of the (Sm,Pr) ${ }_{5} \mathrm{Co}_{19}$ phase," J. Appl. Phys. 67(12), 7510-7513 (1990).

Y. Shen and D.E. Laughlin, "Magnetic effects on the symmetry of CBED patterns of ferromagnetic PrCo5," Philosoph. Mag. Lett. 62(3), 187-193 (1990).

A.G. Khachaturyan and D.E. Laughlin, "Structural transformations during decomposition in Cu-Be alloys," Acta Metall. Mater. 38(10), 1823-1835 (1990).

K. Hono, B. Wong and D.E. Laughlin, "Crystallography of $\mathrm{Co} / \mathrm{Cr}$ bilayer magnetic thin films," J. Appl. Phys. 68(9), 4734-4740 (1990).

S.L. Duan, J.O. Artman, B. Wong and D.E. Laughlin, "The dependence of the microstructure and magnetic properties of $\mathrm{CoNiCr} / \mathrm{Cr}$ thin films on the substrate temperature", IEEE Trans. MAG-26(5), 1587-1589 (1990).

T.L. McDevitt, S. Mahajan, D.E. Laughlin, W.A. Bonner and V.G. Keramidas, "Surface phase separation and ordering in compound semiconductor alloys," Mat. Res. Soc. Symp. Proc., 198, 609-623 (1990).

J.-W. Lee, H.S. Lessure, D.E. Laughlin, M.E. McHenry, S.G. Sankar, J.O. Willis, J.R. Cost and M.P. Maley, "Observation of proposed flux pinning sites in neutron-irradiated $\mathrm{YBa}_{2} \mathrm{Cu}_{3} \mathrm{O}_{7-\mathrm{x}}$," Appl. Phys. Lett. 57(20), 2150-2152 (1990).

Y. Shen and D.E. Laughlin, "Microstructural studies of PrCo5 magnets," J. Magnetism and Magnetic Materials 24, 57-66 (1991).

T.L. McDevitt, S. Mahajan, D.E. Laughlin, F.S. Turco, M.C. Tamargo, M.A. Shahid, W.A. Bonner and V.G. Keramidas, "Microstructural characteristics of phase separated and ordered epitaxial layers of III-V compound semiconductors," Proc. International Conference on Science and Technology of Defect Control in Semiconductors, edited by K. Sumino, Elsevier, 1079-1084, 1990.

J-W. Lee, S-C.N. Cheng, M.H. Kryder and D.E. Laughlin, "The relationship between deposition conditions, microstructure and properties of RE-TM thin films, Proc. Materials Research Society Symposium, Vol. 150, 159-164 (1989).

S. Mahajan, M.A. Shahid and D.E. Laughlin, "Current status of atomic ordering and phase separation in ternary and quaternary III-V compound semiconductors," Institute of Physics Conference Series No. 100: Section 3, presented at Microsc. Semicond. Mater. Conf., Oxford, 10-13 April, 1989, IOP Publishing, Ltd. (1989), 143-153.

N. Mahvan, A.M. Zeltser, D.N. Lambeth, D.E. Laughlin and M.H. Kiyder, "Microstructure and magnetic properties of thin-film Co-Ni-Pt for longitudinal recording," IEEE Trans. MAG 26(5), 2277-2279 (1990). 
K. Rook, A.M. Zeltser, J.O. Artman, D.E. Laughlin, M.H. Kryder and R.M. Chrenko, "Multilayer permalloy films grown by molecular-beam epitaxy," J. Appl. Phys. 69(8), 5670-5672 (1991).

H.L. Ho, S. Mahajan, C.L. Bauer and D.E. Laughlin, "Ordered structures in epitaxial nickel silicide films grown on (111) silicon substrates," Materials Science and Engineering B10, 107-115 (1991).

B.Y. Wong, D.E. Laughlin and D.N. Lambeth, "Investigation of $\mathrm{CoNiCr}$ thin films deposited on [100] and [110] Cr single crystals," IEEE Trans. Mag. 27(6), 4733-4735 (1991)

B.Y. Wong and D.E. Laughlin, "A High-Resolution TEM Investigation of the Interfacial Structure of CoNiCr/Cr Bi-Layer Magnetic Thin Films," Electron Microscopy Society of America, Proceedings of 49th Annual Meeting (1991).

D.E. Laughlin and B.Y. Wong, "The crystallography and texture of Co-based thin film deposited on Cr underlayers," IEEE Trans. Mag. 27(6), 4713-4717 (1991).

T.L. McDevitt, S. Mahajan, D.E. Laughlin, W.A. Bonner and V.G. Keramidas, "Effects of annealing on phase separated microstructures in InGaAsP epitaxial layers," Inst. Phys. Conf. Ser. No 117: Section 7, Proc. Microsc. Semicond. Mater. Conf., Oxford, 1991, 477-483.

T.L. McDevitt, S. Mahajan and D.E. Laughlin, "Two-dimensional phase separation in In $_{1-x} \mathrm{Ga}_{x} A s_{y} P_{1-y}$ epitaxial layers," Phys. Rev. B, 45(12), 6614-6622 (1992).

D.J. Rogers, S. Wang, D.E. Laughlin and M.H. Kryder, "Microstructural investigations of FeN and FeAlN thin films for recording head applications," IEEE Trans. Mag. 28(5), 2418-2420 (1992).

Y. Shen, D.E. Laughlin, T. Shinohara and S. Suwabe, "Microstructural origin of the magnetically degraded layer in Sendust metal-in-gap recording heads," IEEE Trans. Mag. 28(5), 2952-2954 (1992).

Y. Deng, D.N. Lambeth and D.E. Laughlin, "The effects of substrate and bias on $\mathrm{CoNiCr} / \mathrm{Cr}$ thin films," IEEE Trans. Mag. 28(5), 3096-3098 (1992).

Y. Shen, D.E. Laughlin and D.N. Lambeth, "Effects of substrate temperature on magnetic properties of CoCrTa/Cr films," IEEE Trans. Mag. 28(5), 3261-3263 (1992).

Y.C. Feng, D.E. Laughlin and D.N. Lambeth, "Texture of $\mathrm{Cr}$ interlayer in double CoCrTa thin films and effects of interlayer on the magnetic properties," IEEE Trans. Mag. 28(5), 3270-3272 (1992).

J.P. Simmons and D.E. Laughlin, "Ordering and phase separation in FCC/L1 2 alloys," in Kinetics of Ordering Transformations in Metals, Ed. H. Chen and V.K. Vasudevan, The Minerals, Metals \& Materials Society, 1992, 217-225. 
include: magnetic video tape and magneto-optic recording media, hard disk thin film media and the mechanical and chemical interaction of the head with the media, electromagnetic actuators using the new high-performance rare earth-iron based permanent magnets, optical and magnetic sensors for determining position, magnetooptic devices and the synthesis of high frequency ferrites.

\section{Selected Recent Publications:}

E.M.T. Velu and D. N. Lambeth, "High Density Recording on SmCo/Cr Thin Film Media," IEEE Trans. Mag., Vol. 28, No. 5, September 1992.

B. D. Martin and D. N. Lambeth, "Track-Edge Noise Versus Erasure State," IEEE Trans. Mag., Vol. 28, No. 5, September 1992.

Y. C. Feng, D. E. Laughlin, and D. N. Lambeth, "Texture of $\mathrm{Cr}$ Interlayer in Double CoCrTa Thin Films and Effects of Interlayer on the Magnetic Properties," IEEE Trans. Mag, Vol. 28, No. 5, September 1992.

Youping Deng, David N. Lambeth, and David E. Laughlin, "The Effects of Substrate and Bias on $\mathrm{CoNiCr} / \mathrm{Cr}$ Thin Films," IEEE Trans. Mag,, Vol. 28, No. 5, September 1992.

Y. Shen, D. E. Laughlin, and D. N. Lambeth, "Effects of Substrate Temperature on Magnetic Properties of CoCrTa/Cr Films," IEEE Trans. Mag., Vol. 28, No. 5; September 1992.

B. Y. Wong, D. E. Laughlin, and D. N. Lambeth, "Investigation of CoNiCr Thin Films Deposited on [100] and [110] Cr Single Crystals," IEEE Trans. Mag., Vol. 27, No. 6, November 1991.

R. Ranjan, D. N. Lambeth, M. Tromel, P. Goglia, and Y. Li, "Laser Texturing for LowFlying-Height Media," J. Appl, Phys., Vol. 69, No. 8, p. 5745, April 15, 1991.

E.M.T. Velu and D. N. Lambeth, "CoSm-Based High Coercivity Thin Films for Longitudinal Recording," J. Appl. Phys., Vol. 69, No. 8, p. 5175, April 15, 1991.

N. Mahvan, A. M. Zeltser, D. N. Lambeth, D. E. Laughlin and M. H. Kryder, "Microstructure and Magnetic Properties of Thin Film CoNiPt for Longitudinal Recording," IEEE Trans. Mag, Vol. 26, No. 5, p. 2277, September 1990.

D. N. Lambeth, et al., "Surface, Interface, and Thin-Film Magnetism," Materials Reports, L. Mater. Res., Vol. 5, No. 6, p. 1299, June 1990.

T. Lin, R. Alani, and D. N. Lambeth, "Effects of Underlayer and Substrate Texture on Magnetic Properties and Microstructure of a Recording Medium," Journal of Magnetism and Magnetic Materials, Vol. 78, p. 213, 1989. 

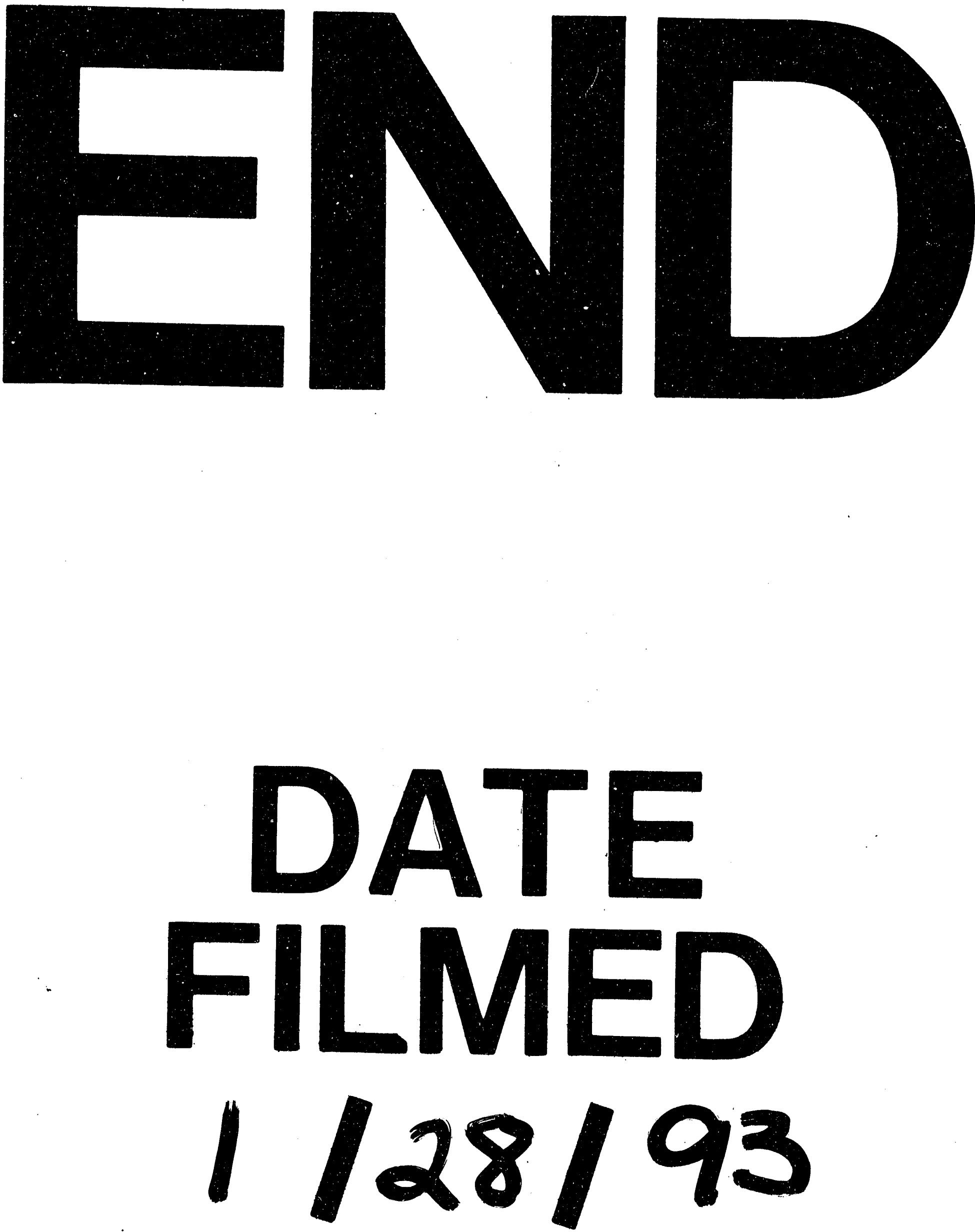
